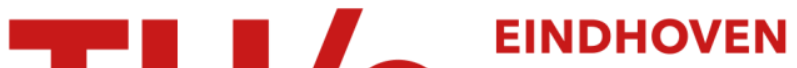 \\ UNIVERSITY OF \\ TECHNOLOGY
}

\section{Spin-polarized tunneling with GaAs tips in scanning tunneling microscopy}

\section{Citation for published version (APA):}

Prins, M. W. J., Jansen, R., \& Kempen, van, H. (1996). Spin-polarized tunneling with GaAs tips in scanning tunneling microscopy. Physical Review B: Condensed Matter, 53(12), 8105-8113.

https://doi.org/10.1103/PhysRevB.53.8105

DOI:

10.1103/PhysRevB.53.8105

Document status and date:

Published: 01/01/1996

\section{Document Version:}

Publisher's PDF, also known as Version of Record (includes final page, issue and volume numbers)

\section{Please check the document version of this publication:}

- A submitted manuscript is the version of the article upon submission and before peer-review. There can be important differences between the submitted version and the official published version of record. People interested in the research are advised to contact the author for the final version of the publication, or visit the $\mathrm{DOI}$ to the publisher's website.

- The final author version and the galley proof are versions of the publication after peer review.

- The final published version features the final layout of the paper including the volume, issue and page numbers.

Link to publication

\section{General rights}

Copyright and moral rights for the publications made accessible in the public portal are retained by the authors and/or other copyright owners and it is a condition of accessing publications that users recognise and abide by the legal requirements associated with these rights.

- Users may download and print one copy of any publication from the public portal for the purpose of private study or research.

- You may not further distribute the material or use it for any profit-making activity or commercial gain

- You may freely distribute the URL identifying the publication in the public portal.

If the publication is distributed under the terms of Article 25fa of the Dutch Copyright Act, indicated by the "Taverne" license above, please follow below link for the End User Agreement:

www.tue.nl/taverne

Take down policy

If you believe that this document breaches copyright please contact us at:

openaccess@tue.nl

providing details and we will investigate your claim. 


\title{
Spin-polarized tunneling with GaAs tips in scanning tunneling microscopy
}

\author{
M. W. J. Prins, * R. Jansen, and H. van Kempen \\ Research Institute for Materials, University of Nijmegen, Toernooiveld 1, NL-6525 ED Nijmegen, The Netherlands
}

(Received 26 July 1995)

\begin{abstract}
We describe a model as well as experiments on spin-polarized tunneling with the aid of optical spin orientation. This involves tunnel junctions between a magnetic material and gallium arsenide (GaAs), where the latter is optically excited with circularly polarized light in order to generate spin-polarized carriers. We present a transport model that takes account of carrier capture in the semiconductor surface states, and describes the semiconductor surface in terms of a spin-dependent energy distribution function. The so-called surface spin splitting can be calculated from the balance of the polarized electron and hole flow in the semiconductor subsurface region, the polarized tunneling current across the tunnel barrier between the magnetic material and the semiconductor surface, and the spin relaxation at the semiconductor surface. We present experimental data obtained in a scanning tunneling microscope with a GaAs tip and a Pt/Co multilayer sample under ambient conditions. A helicity asymmetry of tunnel current (i.e., a circular-polarization-dependent tunnel current) was found with a magnitude of $4 \mathrm{pA}$, which was verified not to be due to variations of the optical power. According to our model and estimations, this observation can be explained by spin-polarized tunneling, with a lower limit to the semiconductor surface spin splitting and spin lifetime of $4 \mathrm{mV}$ and $0.4 \mathrm{~ns}$, respectively.
\end{abstract}

\section{INTRODUCTION}

Since the early seventies, spin-polarized tunneling studies have been conducted in order to derive information about spin-dependent electronic states. ${ }^{1}$ Nearly all of these studies involved thin-film tunnel junctions. With the advent of the scanning tunneling microscope (STM) (Ref. 2) in the eighties, the possibility emerged to perform tunneling studies on a highly localized scale. However, it is nontrivial to develop a spin-selective STM probe (a so-called tunneling tip), that would allow for studies of the spin-dependent electronic structure of magnetic materials with atomic resolution. In this respect, there are essentially three possibilities for the tip material: (i) a superconductor, (ii) a magnetic material, or (iii) a semiconductor. For each of these materials, in this introductory section, we will very briefly point out how spin selectivity can be achieved, and mention the experiments already performed with planar solid-state tunnel junctions as well as in a STM.

(i) The measurement of spin-polarized tunneling with a superconducting material is based on the Zeeman splitting of the (unpaired) quasiparticle states of a spin-paired superconductor (for an excellent review see Ref.1). As a result of an applied magnetic field $H$, in a small spectral range of order $\mu_{B} H$ at the edges of the superconducting gap, tunneling with only one spin orientation is achieved. By measuring the differential conductance versus applied voltage, it is possible to determine the spin polarization of the tunneling current. Many successful experiments were performed with thin-film tunnel junctions involving superconducting $\mathrm{Al}$, an $\mathrm{Al}_{2} \mathrm{O}_{3}$ tunnel barrier, and a wide variety of magnetic counter electrodes. In these junctions, the electron spin is well-conserved during the tunneling process. The application of this technique in a STM is difficult, because a tip with a superconducting state at the apex has to be operated in rather high magnetic fields. To our knowledge, this technique has not yet been applied in a STM.

(ii) In case of tunneling between two magnetic materials, the quantity to be determined is the junction conductance for parallel versus antiparallel orientation of the electrode magnetizations. The relative change of the conductance gives a measure for the product of the spin polarizations of the electrode materials. This technique has been treated in a number of theoretical papers. ${ }^{3}$ Experiments have been performed with planar junctions, ${ }^{4}$ as well as with a STM in an ultrahigh vacuum environment. ${ }^{5}$ Due to magnetostriction and magnetostatic interactions, in a STM the relative orientation of the tip and sample magnetization is not easily modified without changing the tip-to-sample distance. In addition, the perturbation of the sample magnetic structure by the tip remains a matter of concern. It may, however, be possible to obtain some information on spin-polarized tunneling by comparing the surface topography and current-voltage characteristics measured with a magnetic tip on different atomic sites.

(iii) Due to the spin orbit interaction, by optical means a spin selectivity can be achieved in a nonmagnetic semiconductor. For that reason, also a III-V semiconductor material can be used in a spin-polarized tunneling experiment. For example, the injection of nonequilibrium spin-polarized carriers can be detected, due to the emission of polarized radiation. In a STM, the emission of polarized luminescence due to spin-polarized tunneling has already been observed with ferromagnetic tips and a GaAs sample. ${ }^{6}$ On the other hand, spin-polarized carriers can be created by photoexcitation with circularly polarized light, so-called optical spin orientation. ${ }^{7}$ The possibility of using optical spin orientation in GaAs for the purpose of spin-polarized tunneling has been discussed in several publications. ${ }^{8-13}$ These ideas originate from the successful operation of spin-polarized electron sources based on optical excitation of cesium-covered $p$-type GaAs. ${ }^{14}$ In planar $\mathrm{Co} / \mathrm{Al}_{2} \mathrm{O}_{3} / \mathrm{GaAs}$ junctions, evidence for 
spin-polarized tunneling of optically excited electrons was already reported. ${ }^{15,16}$ The application of GaAs for magnetic imaging has become a hot issue with the development of cleaved GaAs tips for STM operation, under ultrahigh vacuum conditions, ${ }^{17}$ as well as under ambient conditions. ${ }^{18,19}$ In the latter studies, optical excitation was already included, but the spin sensitivity of GaAs tips was not yet demonstrated.

In this paper, we are concerned with the usage of optical spin orientation in GaAs in order to achieve spin selectivity in a scanning tunneling microscope. First, a model description of spin-dependent transport will be given, based on the work of Ref. 20. This model includes the subsurface transport processes in the semiconductor and spin relaxation at the semiconductor surface. Experimental results obtained with a GaAs tip on a Pt/Co multilayer sample will be presented. Finally, we will discuss the application of optical spin orientation in GaAs for atomic-scale magnetic imaging.

\section{MODEL FOR SPIN-DEPENDENT TRANSPORT}

In this section, we describe a model for spin-dependent transport in a tunnel junction between a metallic and a semiconductor material, when optical spin orientation is applied. This system is rather complicated, because one should not only consider the tunnel current between the metal and the semicondutor surface, but also the electron and hole currents in the semiconductor subsurface region. The tunneling current has already been treated theoretically by Molotkov ${ }^{11}$ in a Green's function formalism, and by Laiho and Reittu ${ }^{12}$ for plane electronic waves; we will describe the tunneling current by the transfer Hamiltonian approach, ${ }^{21}$ in a convenient form for a modulation experiment. To our knowledge, the spin-dependent transport in the semiconductor, including the subsurface electron and hole currents, the surface states and spin-relaxation therein, has not been treated elsewhere. The incorporation of surface states into a model description is particularly important when considering semiconductor tips, because at the tip apex surface states are present due to the strongly reduced crystallographic symmetry (so even in a well-controlled environment).

In a III-V semiconductor like GaAs, optical spin orientation involves photoexcitation with light of circular polarization. ${ }^{7}$ At the direct gap of GaAs, the conduction band is predominantly formed from $\mathrm{Ga}$-derived wave functions with $s$ symmetry, whereas the valence band consists of Asderived wave functions of $p$ symmetry. Because of the spinorbit interaction in the valence band, the optical transition probabilities are such that circularly polarized photons with an energy close to the band gap give a maximum spin polarization of $50 \%$ in unstrained GaAs. ${ }^{22}$ An important consequence of optical spin orientation, is that in the semiconductor, the energy distribution of charge carriers deviates from equilibrium and is unequal for the two spin orientations, the latter quality being essential for a spin-polarized tunneling experiment.

In the following, we will first consider the spin-dependent tunneling current flowing between a magnetic material and a semiconductor surface, for a given spin-dependent energy distribution of carriers at the semiconductor surface. Next, we will present a model description of the mechanisms that

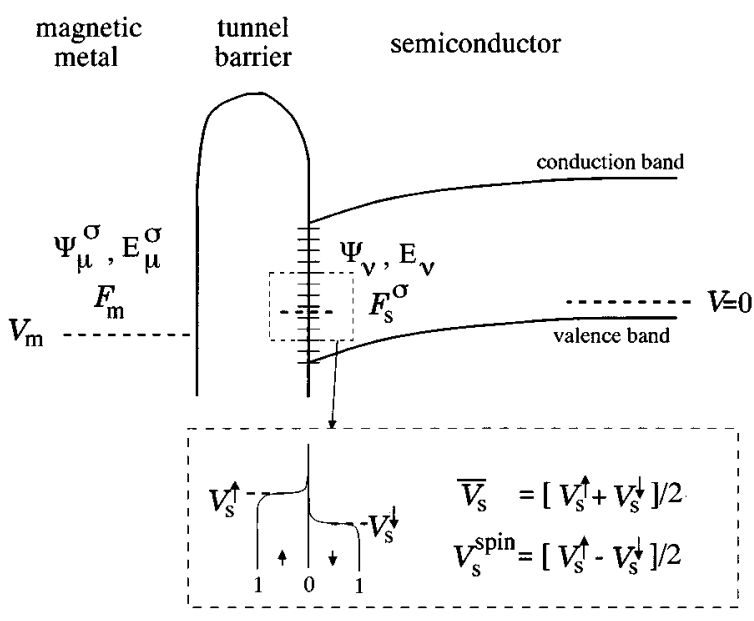

FIG. 1. One-dimensional electronic energy diagram of a tunnel junction between a magnetic metal and a $p$-type semiconductor. The blow up shows the occupation of the surface states for the two spin directions. The spin-dependent quasi-Fermi level is represented by $V_{s}^{\sigma}$. See the text for further explanations. The picture is not on scale, because, in general, the band-bending region in the semiconductor is considerably larger than the tunnel barrier width.

determine the size of the spin dependence of the energy distribution function at the semiconductor surface, the so-called semiconductor surface spin splitting.

\section{A. Spin-polarized tunneling}

The present derivation of the spin-dependent tunneling current is based on the transfer Hamiltonian approach, a firstorder perturbation method that applies in the case of a low tunnel barrier transparency. ${ }^{21}$ As depicted in Fig. 1, the magnetic electrode is described by single-particle spin-dependent wave functions $\psi_{\mu}^{\sigma}$ with energies $E_{\mu}^{\sigma}$, and by a spinindependent energy distribution function $F_{m}$. The $\sigma$ superscript indicates the spin orientation with respect to a given quantization axis (parallel or spin up equals $\uparrow$, antiparallel or spin down equals $\downarrow$ ); we will use identical spin quantization axes for the two materials. Since the semiconductor is not magnetic, the semiconductor surface is described by the spin-independent wave functions $\psi_{\nu}$ with energies $E_{\nu}$; however, due to optical spin orientation, the carriers at the semiconductor surface follow a spin-dependent energy distribution $\left(F_{s}^{\sigma}\right)$.

In a good tunnel barrier no scattering centers are available, such that the electron energy and the electron spin are conserved during the tunneling process. In that case, the tunnel current $\left(I_{t}^{\sigma}\right)$ for spin orientation $\sigma$ from the magnetic material to the semiconductor surface can be expressed as follows:

$$
\begin{array}{r}
I_{t}^{\sigma}=\frac{1}{-e} \int d \varepsilon\left[F_{m}\left(\varepsilon+e V_{m}\right)-F_{s}^{\sigma}(\varepsilon)\right] G_{t}^{\sigma}(\varepsilon), \\
G_{t}^{\sigma}(\varepsilon)=\frac{2 \pi e^{2}}{\hbar} \sum_{\mu \nu} \delta\left(\varepsilon+e V_{m}-E_{\mu}^{\sigma}\right) \delta\left(\varepsilon-E_{\nu}\right)\left|M_{\mu \nu}^{\sigma}\right|^{2},
\end{array}
$$




$$
M_{\mu \nu}^{\sigma}=\frac{\hbar^{2}}{2 m} \int d \vec{S}\left[\psi_{\mu}^{\sigma *} \nabla \psi_{\nu}-\psi_{\nu} \nabla \psi_{\mu}^{\sigma *}\right]
$$

where $e$ is the absolute magnitude of the electron charge. The magnetic electrode is at the externally applied potential $V_{m}$. The energy zero is given by the Fermi level in the semiconductor bulk. The function $G_{t}^{\sigma}(\varepsilon)$ takes account of all energy-conserving tunneling transitions at energy $\varepsilon$, for states with spin orientation $\sigma$. As we will see, $G_{t}^{\sigma}$ closely relates to the differential tunneling conductance. $M_{\mu \nu}^{\sigma}$ is the well-known tunneling matrix element; the surface integral $\left(\int d S\right)$ is evaluated inside the barrier separating the two materials. Note that no assumption is made with respect to the shape of the two electrodes, or the dimensionality of the system. The matrix element takes account of the overlap of the wave functions of the respective electrode materials. This parameter is spin dependent, because the wave functions of the magnetic material depend on the electron spin. Calculation of the total tunnel current yields

$$
I_{t}=\sum_{\sigma} I_{t}^{\sigma}=\frac{1}{-e} \int d \varepsilon\{\underbrace{\left[G_{t}^{\uparrow}+G_{t}^{\downarrow}\right]\left[F_{m}-\bar{F}_{s}\right]}_{\text {spin integrated }}-\underbrace{\left[G_{t}^{\uparrow}-G_{t}^{\downarrow}\right]\left[F_{s}^{\uparrow}-F_{s}^{\downarrow}\right] / 2}_{\text {spin selective }}
$$

where $\bar{F}_{s} \equiv\left[F_{s}^{\uparrow}+F_{s}^{\downarrow}\right] / 2$ is the spin-averaged distribution function at the semiconductor surface. The first term takes account of the spin-integrated tunnel current. The second term is present in the case of a spin-polarized magnetic material $\left(G_{t}^{\uparrow} \neq G_{t}^{\downarrow}\right)$ and a spin imbalance in the semiconductor $\left(F_{s}^{\uparrow} \neq F_{s}^{\downarrow}\right)$. An expression similar to Eq. (4) was derived in Ref. 11. In the following, we will assume that at the semiconductor surface each spin subsystem is close to thermal equilibrium, because the processes of carrier capture and relaxation are generally very efficient at surfaces with surface states. ${ }^{23}$ This means that the spin subsystems approximately follow an energy-shifted Fermi-Dirac distribution: $F_{s}^{\sigma}(\varepsilon) \simeq f\left(\varepsilon+e V_{s}^{\sigma}\right)$, where $V_{s}^{\sigma}$ is the spin-dependent surface potential. As indicated in Fig. 1, this allows for the definition of the spin-averaged surface potential: $\bar{V}_{s} \equiv\left[V_{s}^{\uparrow}+V_{s}^{\downarrow}\right] / 2$; and of the surface spin splitting: $V_{s}^{\mathrm{spin}} \equiv\left[V_{s}^{\uparrow}-V_{s}^{\downarrow}\right] / 2$.

In our experiments a modulation of optical polarization and/or of optical intensity is applied. As a result, the spindependent distribution function at the semiconductor surface becomes time dependent with the following form: $F_{s}^{\sigma}(t)=\operatorname{Re}\left\{F_{s}^{\sigma}+\Delta F_{s}^{\sigma} \exp (j \omega t)\right\}$, where $\omega$ is the modulation frequency. The associated time-dependent surface potential becomes $V_{s}^{\sigma}(t)=\operatorname{Re}\left\{V_{s}^{\sigma}+\Delta V_{s}^{\sigma} \exp (j \omega t)\right\}$. To first order in the modulation amplitudes, with Eq. (4) we find the following modulation of the total tunneling current:

$$
\Delta I_{t}=-\{\underbrace{\left.G_{i}^{\dagger}+G_{i}^{\downarrow}\right] \Delta \bar{V}_{s}}_{\text {spin integrated }}+\underbrace{\left[G_{i}^{\dagger}-G_{t}^{\downarrow}\right] \Delta V_{s}^{\text {spin }}}_{\text {spin selective }}\},
$$

where $\Delta \bar{V}_{s}=\left[\Delta V_{s}^{\uparrow}+\Delta V_{s}^{\downarrow}\right] / 2$ and $\Delta V_{s}^{\mathrm{spin}}=\left[\Delta V_{s}^{\uparrow}-\Delta V_{s}^{\downarrow}\right] / 2$. The spin-selective contribution of Eq. (5) can also be written as follows:

$$
\Delta I_{t}=-G_{t} \mathscr{P}\left(G_{t}\right) \Delta V_{s}^{\mathrm{spin}},
$$

where $G_{t} \equiv G_{t}^{\uparrow}+G_{t}^{\downarrow}$ and $\mathscr{P}\left(G_{t}\right) \equiv\left[G_{t}^{\uparrow}-G_{t}^{\downarrow}\right] / G_{t}$. The negative sign in Eq. (6) results from the definition of the direction of current flow. $\mathscr{P}\left(G_{t}\right)$ is the normalized polarization of the spin-dependent tunnel conductance. This quantity was evaluated by Laiho and Reittu ${ }^{12,13}$ for a two-band free-electron ferromagnet in a planar junction, showing that the size of $\mathscr{P}\left(G_{t}\right)$ depends not only on the bulk band structure, but also on the barrier height and shape. Experiments ${ }^{1}$ as well as calculations ${ }^{12}$ indicate that $\mathscr{P}\left(G_{t}\right)$ can be tens of percents for materials like $\mathrm{Fe}, \mathrm{Co}$, and $\mathrm{Ni}$.

An ideal optical spin-orientation experiment involves only a modulation of the surface spin splitting $\left(\Delta V_{s}^{\text {spin }}\right)$, not a modulation of the spin-averaged surface potential $\left(\Delta \bar{V}_{s}=0\right)$. However, in case of an unwanted modulation of the optical power, the spin-averaged potential will also modulate. ${ }^{24}$ This can, for example, be due to the magnetooptical Kerr/Faraday effect. ${ }^{25}$ Also nonmagnetic effects can give a modulation of optical power, for example, polarization-dependent optical scattering in the tunnel junction, or imperfect alignment of optical components. In our experiments, we find that $\Delta V_{s}^{\text {spin }}$ and $\Delta \bar{V}_{s}$ are of comparable size, namely, of the order of a few percent of $\bar{V}_{s}$, i.e., of the order of $10 \mathrm{mV}$. In order to separate the spin selective from the spin-integrated contributions to the current modulation, an additional technique is required. This separation can be achieved by varying the photon energy, the tunnel barrier width, and the applied voltage. In addition to the abovedescribed modulation of tunnel current, displacement currents and signals due to thermal expansion can appear (see Ref. 20). These signals are not of interest here, since they carry no spin selectivity.

\section{B. Semiconductor surface spin splitting}

The spin dependence of the energy distribution function at the semiconductor surface (the so-called surface spin split- 
ting) is determined by the flow of spin-polarized minority and majority carriers in the semiconductor subsurface region, ${ }^{26}$ the spin-relaxation rate at the semiconductor surface, and the spin-polarized tunnel current from the semiconductor surface to the magnetic electrode. In order to calculate the semiconductor spin splitting, we present a onedimensional spin-dependent transport model. This model is based on the work of Ref. 20, where the electron spin was still ignored. As was already pointed out in Fig. 1, the metalsemiconductor junction is modeled as a device with three "electrodes:" the metallic electrode, the semiconductor surface states, and the semiconductor bulk. The metal is biased with respect to the semiconductor bulk Fermi level by the externally applied voltage $V_{m}$. Between the metal and the surface states, a tunnel barrier is present; the surface states and semiconductor bulk are separated by a Schottky barrier, i.e., the semiconductor subsurface band-bending region. The band-bending region represents a barrier for majority carrier transport; at the same time, it constitutes an accelerating field for photoexcited minority carriers. It was pointed out in Ref. 20 that the deviations from one-dimensional carrier flow in a STM junction can be accomodated by introducing an effective section for minority and majority carrier transport in the semiconductor subsurface region; for clarity, we will omit this effective transport section in the following.

The important spin-polarized currents are given by the current density of photoexcited carriers $\left(J_{p}^{\sigma}\right)$, the majority carrier current density through the Schottky barrier $\left(J_{s}^{\sigma}\right)$, and the tunnel current density $\left(J_{t}^{\sigma}\right)$. We define $J_{t}^{\sigma}$ to flow from the magnetic electrode to the semiconductor surface; $J_{p}^{\sigma}$ and $J_{s}^{\sigma}$ represent flow from the semiconductor surface to the semiconductor bulk. Let the density of spin-up (-down) electrons at the semiconductor surface be given by $N_{\mathrm{ss}}^{\uparrow}\left(N_{\mathrm{ss}}^{\downarrow}\right)$. The excess density of spin-up electrons is defined as $N_{\mathrm{ss}}^{\text {spin }} \equiv$ $N_{\mathrm{ss}}^{\uparrow}-N_{\mathrm{ss}}^{\downarrow}=-e V_{s}^{\mathrm{spin}} D_{\mathrm{ss}}$, where $D_{\mathrm{ss}}$ is the total density of surface states (units $\mathrm{m}^{-2} \mathrm{~J}^{-1}$ ). The density of surface states is assumed to be uniform over the range of interest [as, for example, applies to the native oxide on GaAs (Ref. 27)]. When at the surface the spin lifetime is given by $\tau^{\text {spin }}$, the density of current flowing from the spin-up to the spin-down spin subsystem at the surface becomes

$$
J_{\mathrm{ss}}^{\mathrm{spin}}=\frac{-e N_{\mathrm{ss}}^{\mathrm{spin}}}{\tau^{\mathrm{spin}}}=\frac{e^{2} D_{\mathrm{ss}}}{\tau^{\mathrm{spin}}} V_{s}^{\mathrm{spin}} \equiv \tilde{G}_{\mathrm{ss}}^{\mathrm{spin}} V_{s}^{\mathrm{spin}},
$$

where $\tilde{G}_{\mathrm{ss}}^{\mathrm{spin}} \equiv e^{2} D_{\mathrm{ss}} / \tau^{\text {spin }}$ is the spin conductance between the spin subsystems at the semiconductor surface. The tilde ( $\sim$ ) denotes that the conductance is defined per unit area. Bookkeeping of the flow of spin and charge yields the following equations for the spin-dependent current densities at the semiconductor surface:

$$
J_{p}^{\uparrow}+J_{s}^{\uparrow}-J_{t}^{\uparrow}+J_{\mathrm{ss}}^{\mathrm{spin}}=0, \quad J_{p}^{\downarrow}+J_{s}^{\downarrow}-J_{t}^{\downarrow}-J_{\mathrm{ss}}^{\mathrm{spin}}=0 .
$$

Adding and substracting these equations, and using the normalized spin polarizations of the respective currents, we find

$$
\begin{gathered}
J_{p}+J_{s}-J_{t}=0, \\
J_{p} \mathscr{P}\left(J_{p}\right)+J_{s} \mathscr{P}\left(J_{s}\right)-J_{t} \mathscr{P}\left(J_{t}\right)+2 \tilde{G}_{\mathrm{ss}}^{\mathrm{spin}} V_{s}^{\mathrm{spin}}=0,
\end{gathered}
$$

where $J_{i} \equiv J_{i}^{\uparrow}+J_{i}^{\downarrow}$ and $\mathscr{P}\left(J_{i}\right) \equiv\left[J_{i}^{\uparrow}-J_{i}^{\downarrow}\right] / J_{i}, i \in\{p, s, t\}$. The so-called photoamperic mode of operation ${ }^{20}$ of the metalsemiconductor tunnel junction refers to the situation that the tunnel barrier represents a far higher conductance than the Schottky barrier, i.e., $J_{t} \simeq J_{p}$, and $\left|J_{s}\right| \ll\left|J_{p}\right|$. In that limit, the size of the tunneling current $\left(J_{t}\right)$ is given by the size of the photocurrent $\left(J_{p}\right)$, and is not affected by the polarization of the photocurrent $\mathscr{P}\left(J_{p}\right)$. Or to put it differently, whatever the polarization of the photocurrent, all the photoexcited carriers will be tranported into the metallic electrode, because the Schottky barrier is too high. This is an undesirable situation for a spin-polarized tunneling experiment, where spin sensitivity is wanted in the total tunneling current. In order to maximize the spin sensitivity in the total tunneling current, we should operate in the so-called photovoltaic regime, ${ }^{20}$ when the tunnel barrier conductance is lower than the conductance of the Schottky barrier. Then the tunnel current $\left(J_{t}\right)$ is negligible with respect to the photocurrent, and $J_{s} \simeq-J_{p}$. This situation has the important advantange that the spin splitting at the semiconductor surface is only determined by the photocurrent and the Schottky majority carrier current, independent of the tunneling current properties.

Let us calculate the size of the spin splitting at the semiconductor surface for p-type GaAs in the photovoltaic mode of operation. By optical spin orientation in GaAs, the spins of the electrons as well as the hole spins are oriented in principle. However, due to the spin orbit interaction, a strong coupling exists between the hole's angular momentum and its quasimomentum $(\vec{k})$, resulting in a loss of the hole spin orientation on the time scale of the momentum relaxation time $\left(\tau_{p} \sim 10^{-13} \mathrm{~s}\right)$; in the conduction band this strong coupling is absent, causing the electron spin lifetime to be many orders of magnitude larger. ${ }^{28}$ In $p$-type GaAs, the bands are generally bending downward from the bulk toward the surface, which drives the optically oriented electrons toward the surface. ${ }^{29}$ In the photovoltaic mode of operation, this flow of electrons (the minority carrier current $J_{p}$ ) is balanced by the hole current that flows through the Schottky barrier (the majority carrier current $J_{s}$ ); the latter is given by thermally assisted transport over the electrostatic barrier and subsequent surface recombination. ${ }^{30,31}$ Since the holes are hardly polarized, we can neglect the spin dependence of the hole energy distribution in the semiconductor bulk; in addition, for a small spin splitting, the surface recombination velocity does not depend on the electron spin. In other words, in $p$-type GaAs we can to the first order neglect the polarization of the majority carrier current $\mathscr{P}\left(J_{s}\right)$, with respect to the polarization of the minority carrier current $\mathscr{P}\left(J_{p}\right)$; In that case, using Eqs. (7) and (9) in the photovoltaic mode of operation $\left(J_{t}=0\right)$, we find the following expression for the spin splitting of the surface potential:

$$
V_{s}^{\mathrm{spin}}=-\mathscr{P}\left(J_{p}\right) J_{p} \frac{\tau^{\mathrm{spin}}}{2 e^{2} D_{\mathrm{ss}}}
$$

Thus, for a maximum spin-selective tunneling current [cf. Eq. (6)], it is appropriate to use $p$-type GaAs in the photovoltaic mode of operation, with a large magnitude and polarization of the photocarrier current, a large surface spin lifetime, and a low density of surface states. 
In summary, we have analyzed the spin-dependent tunneling current in a tunnel junction between a magnetic material and a semiconductor, where in the latter a modulation of spin orientation was established by optical means. In the semiconductor, account was taken of the polarized hole and electron currents, and the carrier capture and transport in the surface states. For future directions, it will be of interest to model the polarization of the majority carrier current, which will be important for large values of the surface spin splitting and for $n$-type materials. ${ }^{29}$

\section{EXPERIMENT}

\section{A. Introduction}

A model experiment on optical spin orientation in a STM can, in principle, be performed with a magnetic sample and a semiconductor tip, or with a semiconductor sample and a magnetic tip. It is important that radiation of near-circular polarization is absorbed near the STM tunneling point. Hence, an illumination geometry that approaches rotational symmetry is preferred, which is best achieved with illumination along the sample normal. This can involve the usage of a semiconductor membrane and a magnetic tip, ${ }^{32}$ or a semitransparent magnetic thin film and a semiconductor tip. The following sections deal with our experiments on a semitransparent $\mathrm{Pt} / \mathrm{Co}$ multilayer and $p$-type GaAs tips.

As was pointed out with Eq. (5), when applying a modulation technique for spin-polarized tunneling by optical spin orientation, one should separate the spin-integrated and spinselective contributions to the current modulation. The spinintegrated contribution can be caused by several effects: (i) Because the junction contains a magnetic material, a helicitydependent optical transmission occurs due to magnetooptical effects such as magnetic circular dichroism. ${ }^{33}$ (ii) If the geometry of the tip/sample combination is of low symmetry, the optical scattering and absorption in the tunnel junction can, in principle, depend on the helicity of the light; this is a near-field optical phenomenon that is hard to control. (iii) Finally, the modulation of optical power can be due to imperfect optical alignment, causing the optical beam that enters the STM to contain a small power modulation in addition to the helicity modulation.

In previously reported experiments with planar junctions, ${ }^{15}$ a variation of photon energy and sample magnetization were employed in order to separate the spinaveraged and spin-selective signals. However, in a STM experiment, it is not trivial to employ these techniques without disturbing the critical optical alignment or the tunnel junction itself. On the other hand, in a metal-semiconductor STM junction there is a strong voltage dependence of the sensitivity of the current to variations of the optical power (see Ref. 20 ); by sweeping the voltage, we can tune the tunnel junction to a low sensitivity to variations of the optical power, such that we can isolate a signal due to spin-polarized tunneling. The involved dual-frequency modulation technique and experimental results are presented in the following sections.

\section{B. Experimental arrangement}

The experiments were performed in a STM at ambient temperature and pressure, in an arrangement similar to the one discussed in Ref. 20. The optical beam of a linearly polarized single-mode $\mathrm{HeNe}$ laser $(633 \mathrm{~nm})$ was guided through a Pockels cell and a polarizer, which provided an intensity modulation (IM) of a few percent at $80 \mathrm{kHz}$. The beam passed through a photoelastic modulator (PEM), introducing a 84-kHz sinusoidal polarization modulation (PM) between a positive and negative helicity of the light. Thereafter, the beam was focused onto the tunnel junction by a $30-\mathrm{mm}$ focal length objective, to a spot of $20 \pm 5 \mu \mathrm{m}$ diameter. The tunneling current $\left(I_{t}\right)$ was measured by a homemade $100-\mathrm{mV} / \mathrm{nA}$ current-to-voltage converter with a bandwidth of about $100 \mathrm{kHz}$. Above $\sim 60 \mathrm{kHz}$ the converter response was determined with an estimated accuracy of $10 \%$ and a phase uncertainty of about $10^{\circ}$. The bandwidth of the STM constant-current regulation system was $2 \mathrm{kHz}$. The current signal was fed into two lock-in amplifiers for phasesensitive detection of the current modulation $\left(\Delta I_{t}\right)$ at 80 and $84 \mathrm{kHz}$. A signal of spin-polarized tunneling due to optical spin orientation appears in the PM signal (circular polarization modulation at $84 \mathrm{kHz}$ ). As was pointed out above, in the PM signal there can also be a contribution due to a simultaneously occuring unwanted modulation of the optical intensity at $84 \mathrm{kHz}$. We employ the IM signal (intensity modulation at $80 \mathrm{kHz}$ ) in order to determine the sensitivity of the tunnel current to a modulation of optical intensity. This knowledge can serve to isolate the contribution due to spinpolarized tunneling in the PM signal at $84 \mathrm{kHz}$ (circular polarization modulation). The frequencies of the modulations ( 80 and $84 \mathrm{kHz}$ ) were chosen of similar magnitude in order to ascertain an equal response of the total current detection system.

The GaAs tips were prepared by cleaving (001) wafers along (110) and $(1 \overline{1} 0)$ directions, forming a corner bounded by these planes. Inspection by scanning electron microscopy and the STM showed that cleavage produces well-defined corners with tip apex radii smaller than $100 \mathrm{~nm}$. The $\mathrm{Pt} / \mathrm{Co}$ multilayer sample consists of a $6-\AA$ Pt base layer and 20 pairs of $3.5-\AA$ Co and $6-\AA$ Pt layers evaporated on a glass substrate. ${ }^{34}$ This material exhibits perpendicular magnetic anisotropy with $99 \%$ magnetic remanence. No external magnetic field was applied during the measurements.

\section{Results}

Figure 2 shows static (so with the tip-sample distance regulation system turned off) curves of the current modulations measured by the lock-in amplifiers (top panel) and the measured tunneling current (bottom panel) versus applied voltage, for a $p$-type GaAs tip of $10^{25} \mathrm{~m}^{-3}$ doping density. The indicated data are averages of 225 spectroscopic curves, each curve taken in $60 \mathrm{~ms}$ time. In the bottom panel, the zero-current point is clearly displaced along the voltage axis, indicating the presence of a photoinduced surface voltage of about $-0.1 \mathrm{~V}$. The current modulations were detected in phase with the modulations applied to the optical beam. The square symbols represent the signal due to a modulation of the optical intensity. At reverse bias (positive sample voltage), the current is most sensitive to intensity variations, whereas in forward bias (negative sample voltage), the sensitivity is small. At even higher forward bias, the modulation signal reverses sign. These observations are in agreement 

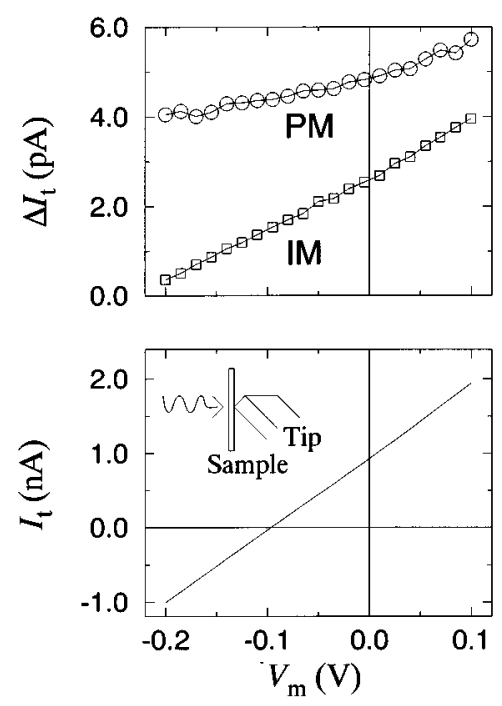

FIG. 2. Experimental results of the dual-frequency modulation technique applied to a $p$-type GaAs tip of $10^{25} \mathrm{~m}^{-3}$ doping density tunneling on a Pt/Co multilayer. The indicated data are averages of 225 spectrocopic curves, each curve taken in $60 \mathrm{~ms}$ time. Top panel: Current modulations measured by the lock-in amplifiers, as a function of the voltage applied to the magnetic sample $\left(V_{m}\right)$. The square symbols indicate the signal resulting from a modulation of the optical intensity (IM). The circular symbols denote the signal due to a modulation of the light polarization (PM). The estimated signal uncertainty, due to drift during the measurements, is about $1 \mathrm{pA}$. Bottom panel: Measured current versus voltage curve for the same junction. The inset shows the illumination geometry. Incident optical power $P=5 \mathrm{~mW}$.

with the model and experimental results reported in Ref. 20. The circular symbols represent the signal measured at the frequency of circular PM. Clearly this curve has a different voltage dependence than the curve of intensity modulation. In particular, where the sensitivity to intensity modulation tends to zero (at $V_{m} \simeq-0.2 \mathrm{~V}$ ), still a significant helicitydependent current is observed of size $\Delta I_{t}=4 \pm 1 \mathrm{pA}$. To the first order ${ }^{35}$ we can attribute this current modulation to spindependent tunneling of optically oriented charge carriers. The data in Fig. 2 represent the clearest indication that we obtained on the possibility of spin-polarized tunneling by optical spin orientation in a STM. It was not possible to perform a similar measurement while scanning, due to the increased noise levels and shorter measurement times.

\section{DISCUSSION}

With the aid of our model on spin-polarized transport, in the following we calculate the semiconductor spin splitting and the surface spin lifetime from estimations of the spin polarization of the tunneling conductance and of the photocurrent.

Tunneling conductance polarization. In order to estimate the spin splitting at the semiconductor surface, we need to assess the spin polarization of the tunnel conductance $\mathscr{P}\left(G_{t}\right)$ for our Pt/Co multilayer. In earlier spin-polarized measurements with thin-film tunnel junctions, for Co a spin polarization of $\left|\mathscr{P}\left(G_{t}\right)\right|=0.35$ was detected. ${ }^{1}$ However, for several reasons we cannot simply adopt this value for our sample surface.

(i) The Pt/Co multilayer was capped with $6 \AA$ of Pt, i.e., with $2-3$ atomic layers of Pt. This means that the electron tunneling does not occur directly into the Co, but rather into the Pt surface atoms that are spin polarized, due to the exchange interaction with the underlying Co. ${ }^{36}$

(ii) We do not expect the polarization of the electronic states to be uniform over the sample surface, because the $\mathrm{Pt} / \mathrm{Co}$ multilayer is constituted of microcrystallites with a diameter of 10 to $20 \mathrm{~nm},{ }^{34}$ as we also verified with STM measurements.

(iii) Due to the operation under ambient conditions, a thin contamination layer will have formed on the surface. It is well known that tunneling is not inhibited by a contamination layer, and that $\mathrm{Pt} / \mathrm{Co}$ multilayers are resistant to oxidation. ${ }^{34}$ Nevertheless, the operation under ambient conditions adds uncertainty to the value of the surface polarization. From the previous statements, we believe that the average polarization of our sample surface should be more than an order of magnitude lower than of pure Co; however, at selected sites, we assume that a reasonable upper limit for the polarization $\left|\mathscr{P}\left(G_{t}\right)\right|$ is about 0.1 .

With this estimate, we deduce the semiconductor surface spin splitting in the following way. From the $I$ - $V$ curve depicted in Fig. 2, we deduce a tunnel barrier conductance of $G_{t} \simeq 10^{-8} \Omega^{-1}$; using Eq. (6), the measured tunnel current modulation of $4 \mathrm{pA}$, and the estimated upper limit for $\left|\mathscr{P}\left(G_{t}\right)\right|$ of 0.1 , we deduce that, in our experiment, the value for the spin splitting $\left(\Delta V_{s}^{\text {spin }}\right)$ was $4 \mathrm{mV}$ or larger (we pay no attention to the sign of the polarization, since we did not calibrate the sign of the helicity dependence of the current). With a measured surface photovoltage of $0.1 \mathrm{~V}$ (cf. Fig. 2), we obtain a relative spin splitting of $\left|\Delta V_{s}^{\mathrm{spin}} / \bar{V}_{s}\right|=(4 \pm 3)$ $\times 10^{-2}$ or larger. Note that this value is close to the experimental result ${ }^{16}$ obtained with planar junctions of $\mathrm{Al}_{2} \mathrm{O}_{3}$ and Co.

Photocurrent polarization. The polarization of the photoexited minority carrier current $\mathscr{P}\left(J_{p}\right)$ arriving at the surface of our GaAs tip is determined by the magnitude of the spin polarization at excitation and the spin relaxation during transport to the semiconductor surface. In addition, optical refraction in the GaAs tip may reduce the photocurrent polarization. These issues are addressed in the following.

(i) Theoretically, the absorption of circularly polarized gap-energy photons gives an electron spin polarization of 0.5 in GaAs. ${ }^{22}$ Between creation and arrival at the semiconductor surface, the photoexcited carriers suffer energy and spin relaxation. For this reason, the spin polarization of electrons photoemitted from GaAs (doping $\sim 10^{25} \mathrm{~m}^{-3}$ ) that is activated to negative electron affinity, usually ranges from 0.2 to 0.3 at room-temperature operation. ${ }^{37}$ For photons with an energy larger than the band gap, the theoretical value of the spin polarization at excitation is reduced and the carriers suffer additional energy and spin relaxation before arrival at the semiconductor surface. For a photon energy of $1.96 \mathrm{eV}$ (633 $\mathrm{nm}$ wavelength), the spin polarization of photoemitted electrons decreases by more than a factor of three when compared to the case where gap-energy $(1.42 \mathrm{eV})$ photons are used. $^{37,38}$

(ii) The optical polarization inside the GaAs is affected by 
the shape of the material. A rigorous calculation of the polarization-dependent penetration of optical waves into a semiconductor tip is complicated by the nontrivial geometry of the STM junction. By a simple calculation however, we can estimate an upper limit to the distortion of the optical polarization in the tunnel junction. Ideally, our cleaved GaAs tunneling tips have the shape of a regular tetrahedron, i.e., at the apex there is a threefold rotational symmetry around the tip axis. If the tip axis is collinear with the optical propagation direction, then at the tip apex there is no asymmetry to linear or circular polarization; in other words, the polarization of the impinging light is conserved along the tip axis in the semiconductor material. However, because in a STM experiment also lateral minority carrier transport is involved (see Ref. 20 and the notion of an effective collection radius), the off-axis optical polarization is of importance. Off the tip axis, the local geometry is determined by the tip surface, of which the normal vector is at a large angle with the optical propagation direction: for our cleaved tips this angle amounts to $45^{\circ}$. We can easily calculate the optical penetration of a circularly polarized plane wave at an angle of $45^{\circ}$ into GaAs,${ }^{39}$ which shows that the reduction of the degree of circular polarization in the GaAs is only $12 \%$.

(iii) When in GaAs circularly polarized photons with near-band-gap energy are absorbed, at a fixed location the average spin orientation of the photoexcited electrons is normal to the plane of rotation of the electric vector, i.e., collinear with the optical propagation direction. ${ }^{7}$ However, due to the optical refraction the propagation direction is not uniform in the GaAs tip. For a GaAs surface at an angle of $45^{\circ}$, the propagation direction of the transmitted beam is modified by an angle of $45^{\circ}-11^{\circ}=34^{\circ}$, with respect to the incident beam. ${ }^{39}$ In our experiment, the propagation direction of the incident beam (i.e., the sample normal) is a convenient spin quantization axis, because the $\mathrm{Pt} / \mathrm{Co}$ multilayer sample has its magnetic anisotropy along the same direction. In other words, due to the optical refraction the direction of the spin orientation of photoexcited carriers deviates at most $34^{\circ}$ from the quantization axis. Projection of the direction of the spin polarization on the quantization axis gives a factor of $\cos \left(34^{\circ}\right)=0.83$, i.e., the modified optical propagation direction results in a reduction of the spin polarization by $17 \%$. In conclusion, from statements (i)-(iii), we deduce that a value of 0.1 represents an upper limit to the photocurrent polarization for our experimental situation.

With the previously deduced value of the spin splitting $\left(V_{s}^{\text {spin }} \geqslant 4 \mathrm{mV}\right)$, we calculate the spin lifetime by using Eq. (10) of our model description. In our experiment, the illumination intensity was about $10^{7} \mathrm{Wm}^{-2}$, which implies that $J_{p} \simeq 5 \times 10^{6} \mathrm{Am}^{-2}$ (see Ref. 20); the density of surface states $\left(D_{\mathrm{ss}}\right)$ in the native oxide on GaAs is $10^{36} \mathrm{~m}^{-2} \mathrm{~J}^{-1}$ or larger. ${ }^{27,31}$ Using these values and $\mathscr{P}\left(J_{p}\right) \leqslant 0.1$, with Eq. (10) we deduce that the surface spin lifetime ( $\left.\tau^{\text {spin }}\right)$ was $0.4 \mathrm{~ns}$ or larger in our experiment.

Concerning the application of optical spin orientation to magnetic imaging, it is of interest to estimate the importance of magnetic forces. In our experiments, the maximum light intensity was of the order of $10^{7} \mathrm{Wm}^{-2}$. For $1.5 \mathrm{eV}$ photons the absorption depth is about $1 \mu \mathrm{m}$ in $\mathrm{GaAs} ;{ }^{41}$ if every photon yields one electron-hole pair, the photoelectron generation rate is $4 \times 10^{31}$ electrons per second per $\mathrm{m}^{3}$. In the case of $50 \%$ spin polarization and a lifetime of $10 \mathrm{~ns}$, in the semiconductor this gives a maximum photoinduced magnetization of $2 \times 10^{23} \mu_{B} \mathrm{~m}^{-3}$. This is equal to an average optically induced magnetic moment of less than $10^{-5} \mu_{B}$ per atom. With such a low magnetization the resulting magnetic dipolar forces are negligibly small. It is more difficult to evaluate the size of an exchange force in an optically oriented metalsemiconductor tunnel junction, because of the nonequilibrium spin dynamics involved. Concerning the spin splitting at the semiconductor surface, from our experimental results we deduce that the spin splitting $V_{s}^{\text {spin }}(4 \mathrm{mV})$ is smaller than the surface photovoltage $(0.1 \mathrm{~V})$. Although a spin splitting as high as $0.1 \mathrm{~V}$ may be achievable, this is still an order of magnitude smaller than the exchange splitting in Fe or Co. From the above estimates we conclude that the magnetic forces, in a tunnel junction between a ferromagnetic material and an optically excited semiconductor, are small compared to the case of two magnetic counter electrodes.

\section{CONCLUSIONS}

We presented a dual-frequency modulation technique that allows for the detection of a spin-polarized tunneling signal, due to optical spin orientation in a STM configuration. A modulation of optical polarization not only generates a modulation of spin orientation, but concurrently gives a modulation of the amount of photoexcited carriers. This can be caused by the magneto-optical Kerr/Faraday effect in the magnetic material, a low symmetry of the tunnel junction, or improper optical alignment. Thus, for the detection of spinpolarized tunneling it is a prerequisite to tune the tunnel junction to a low sensitivity for unwanted variations of the optical intensity. In order to find the required parameters (e.g., applied voltage, tip-sample separation), we have to analyze the sensitivity to intensity variations by modulating the optical intensity (at a different frequency than the modulation of optical polarization). In a metal/semiconductor STM junction, this sensitivity strongly depends on the applied voltage. By sweeping the applied voltage one can conveniently select the junction parameters such that spinpolarized tunneling can be detected.

Using a GaAs tip and a Pt/Co multilayer under ambient conditions, we delivered evidence for the possibility of spinpolarized tunneling by optical spin orientation. The measured tunnel current modulation due to spin-polarized tunneling was $4 \mathrm{pA}$. The estimated spin polarization of the tunneling conductance is 0.1 or lower in that experiment; with this estimation, the experimental observation implies that the spin splitting at the semiconductor surface $\left(V_{s}^{\text {spin }}\right)$ was $4 \mathrm{mV}$ or larger. According to our model and estimations, a spin splitting of that size corresponds to a surface spin lifetime of $0.4 \mathrm{~ns}$ or larger.

Although we were not able to perform magnetic imaging, this should be possible by increasing the ratio of signal to noise; this may involve an optimization of the photon energy, the optical intensity, and the semiconductor surface preparation. Furthermore, it will be of importance to improve our knowledge of the spin polarization in the semiconductor and in the magnetic material. With respect to the semiconductor, information on the polarization of the photoexcited carriers can be derived from measurements of polarized photolumi- 
nescence or spin-polarized photoemission; an improved definition of the surface electronic properties of the magnetic material can be achieved by preparation and in situ measurement of the sample in a well-controlled environment, such as an ultrahigh vacuum.

\section{SUMMARY}

In this paper, we presented a model for spin-polarized transport in a photoexcited tunnel junction between a magnetic metal and a semiconductor, where the semiconductor charge carriers are polarized by optical orientation. The semiconductor surface was described in terms of a spindependent distribution function or quasi-Fermi level $V_{s}^{\sigma}$. The model clearly demonstrates that the spin-selective contribution to the total tunneling current is proportional to the product of the spin dependence of the tunneling conductance $\left(G_{t}^{\uparrow}-G_{t}^{\downarrow}\right)$ and the spin-split quasi-Fermi level at the semiconductor surface $\left(V_{s}^{\uparrow}-V_{s}^{\downarrow}\right)$. The sensitivity of the total tunneling current is maximized in the photovoltaic mode of operation, i.e., when the tunnel current represents a negligible disturbance to the semiconductor. For that mode of operation, in a $p$-type material the spin splitting at the semiconductor surface is proportional to the density and the polarization of the photoexcited electrons, to the surface spin lifetime, and inversely proportional to the density of surface states.

We used a GaAs tip and a Pt/Co multilayer in a STM configuration. A technique of polarization modulation was employed, and the resulting tunnel current modulation was detected. A complication is that the current modulation is caused by optical spin-orientation and spin-dependent transmission over the tunnel barrier, as well as by a modulation of optical power absorbed in the semiconductor. The power modulation can, for example, be due to the magneto-optical Kerr/Faraday effect. The separation of the two contributions can be established by varying the photon energy, the tunnel barrier width, and as shown here by adjusting the applied voltage. From the experiment it was deduced that the relative spin splitting $\left(\left[V_{s}^{\uparrow}-V_{s}^{\downarrow}\right] /\left[V_{s}^{\uparrow}+V_{s}^{\downarrow}\right]\right)$ at the semiconductor surface was $4 \%$ or larger; according to our model this observation corresponds to a spin lifetime of 0.4 ns or larger.

Summarizing, optical spin orientation in III-V semiconductors provides a unique opportunity to detect spinpolarized tunneling in a STM. This is mainly due to the fact that the spin polarization in the semiconductor can be reversed by optical means. We successfully investigated this mechanism for spin selectivity, in a STM with a cleaved GaAs tip. In an ultrahigh vacuum environment, with cleaved GaAs tips atomic imaging of a nonmagnetic material was already demonstrated. ${ }^{17}$ We believe that magnetic imaging with subnanometer resolution is within reach, using optically oriented semiconductor tips in a STM. As was already demonstrated in Ref. 25, with a similar experimental arrangement it is possible to perform magneto-optical near-field imaging. Magneto-optical interactions are sensitive to bulk magnetization, whereas spin-polarized tunneling is sensitive to the spin dependence of the electron states at the sample surface. The respective effects may be separated by their dependence on photon energy, tunnel barrier width, and bias voltage. Ideally, one would like to combine the two measurements, so as to be able to simultaneously measure sample topography, bulk magnetization, and surface spin structure with (sub-)nanometer resolution.

\section{ACKNOWLEDGMENTS}

We thank J. Hermsen, J. Gerritsen, and A. van Etteger for technical support. H. van Kesteren, H. Donkersloot, and J. Kerkhof of Philips Research Laboratories prepared the Pt/Co multilayers. A. van Geelen supplied us with semiconductor materials. We thank H. Salemink and R. de Groot for critically reading the manuscript. Part of this work was supported by the Stichting Fundamenteel Onderzoek der Materie (FOM), which is financially supported by the Nederlandse Organisatie voor Wetenschappelijk Onderzoek (NWO).
*Present address: Philips Research Laboratories, Prof. Holstlaan 4, 5656 AA Eindhoven, The Netherlands.

${ }^{1}$ R. Meservey and P.M. Tedrow, Phys. Rep. 238, 173 (1994).

${ }^{2}$ A review on STM can be found in Scanning Tunneling Microscopy I, edited by R. Wiesendanger and H.-J. Güntherodt, Springer Series in Surface Science Vol. 20 (Springer Verlag, Heidelberg, 1992); Scanning Tunneling Microscopy II, edited by R. Wiesendanger and H.-J. Güntherodt, Springer Series in Surface Science Vol. 28 (Springer Verlag, Heidelberg, 1993); Scanning Tunneling Microscopy III, edited by R. Wiesendanger and H.-J. Güntherodt, Springer Series in Surface Science Vol. 29 (Springer Verlag, Heidelberg, 1993).

${ }^{3}$ J.C. Slonczewski, Phys. Rev. B 39, 6995 (1989); A.A. Minakov and I.V. Shvets, Surf. Sci. Lett. 236, L377 (1990); S.N. Molotkov, Surf. Sci. 261, 7 (1992); D. Bürgler and G. Tarrach, Ultramicroscopy 42, 194 (1992).

${ }^{4}$ J.S. Moodera, L.R. Kinder, T.M. Wong, and R. Meservey, Phys. Rev. Lett. 74, 3273 (1995); T. Miyazaki and N. Tezuka, J. Magn. Magn. Mater. 139, L231 (1995) and references therein.

${ }^{5}$ R. Wiesendanger, H.-J. Güntherodt, G. Güntherodt, R.J. Gambino, and R. Ruf, Phys. Rev. Lett. 65, 247 (1990).
${ }^{6}$ S.F. Alvarado and Ph. Renaud, Phys. Rev. Lett. 68, 1387 (1992).

${ }^{7}$ Optical Orientation, edited by F. Meier and B.P. Zakharchenya, Modern Problems in Condensed Matter Sciences Vol. 8 (Elsevier, Amsterdam, 1984).

${ }^{8}$ Japanese Patent No. A62 139240 (22 June 1987); European Patent No. A0 355241 (28 February 1990).

${ }^{9}$ D.T. Pierce, Phys. Scr. 38, 291 (1988).

${ }^{10}$ W.T.M. Wolters, Master's thesis, University of Nijmegen, 1988.

${ }^{11}$ S.N. Molotkov, Pis'ma Zh. Eksp. Teor. Fiz. 55, 180 (1992) [JETP Lett. 55, 173 (1992)].

${ }^{12}$ R. Laiho and H.J. Reittu, Surf. Sci. 289, 363 (1993).

${ }^{13}$ H.J. Reittu, J. Phys. Condens. Matter 6, 1847 (1994).

${ }^{14}$ C. Hermann, H.-J. Drouhin, G. Lampel, Y. Lassailly, D. Paget, J. Peretti, R. Houdre, F. Ciccacci, and H. Riechert, in Spectroscopy of Nonequilibrium Electrons and Phonons, edited by C.V. Shank and B.P. Zakharchenya (Elsevier, Amsterdam, 1992), Chap. 9, p. 397.

${ }^{15}$ M.W.J. Prins, D.L. Abraham, and H. van Kempen, J. Magn. Magn. Mater. 121, 109 (1993); Surf. Sci. 287/288, 750 (1993).

${ }^{16}$ M.W.J. Prins, H. van Kempen, H. van Leuken, R.A. De Groot, W. Van Roy, and J. De Boeck, J. Phys. Condens. Matter 7, 9447 
(1995); M.W.J. Prins, Ph.D. thesis, University of Nijmegen, 1995.

${ }^{17}$ G. Nunes and N.M. Amer, Appl. Phys. Lett. 63, 1851 (1993).

${ }^{18}$ M.W.J. Prins, M.C.M.M. van der Wielen, R. Jansen, D.L. Abraham, and H. van Kempen, Appl. Phys. Lett. 64, 1207 (1994).

${ }^{19}$ R. Jansen, M.C.M.M. van der Wielen, M.W.J. Prins, D.L. Abraham, and H. van Kempen, J. Vac. Sci. Technol. B 12, 2133 (1994).

${ }^{20}$ M.W.J. Prins, R. Jansen, R.H.M. Groeneveld, A.P. van Gelder, and H. van Kempen, preceeding paper, Phys. Rev. B 53, 8090 (1996); M.W.J. Prins, Ph.D. thesis, University of Nijmegen, 1995.

${ }^{21}$ N.D. Lang, in Scanning Tunneling Microscopy III, edited by R. Wiesendanger and H.-J. Güntherodt, Springer Series in Surface Science Vol. 29 (Springer Verlag, Heidelberg, 1993), Chap. 2.

${ }^{22}$ D.T. Pierce and R.J. Celotta, in Optical Orientation (Ref. 7), Chap. 6.

${ }^{23}$ R.K. Ahrenkiel, in Minority Carriers in III-V Semiconductors: Physics and Applications, edited by R.K. Ahrenkiel and M.S. Lundstrom, Semiconductors and Semimetals Vol. 39 (Academic Press, San Diego, 1993), Chap. 2, p. 39.

${ }^{24} \mathrm{~A}$ modulation of optical power causes a nonzero $\Delta \bar{V}_{s}$, but also affects the value of $\Delta V_{s}^{\text {spin }}$. However, the relative change of the value of $\Delta V_{s}^{\text {spin }}$ is only of the order of $\Delta \bar{V}_{s} / \bar{V}_{s}$, which is negligible in our experiments.

${ }^{25}$ Using this effect, magneto-optical imaging in the near field can be achieved, as shown in M.W.J. Prins, R.H.M. Groeneveld, D.L. Abraham, H. van Kempen, and H.W. van Kesteren, Appl. Phys. Lett. 66, 1141 (1995).

${ }^{26}$ Note that in a semiconducting material, it is important to discriminate between majority and minority carriers (holes or electrons). In magnetic materials it is often useful to distinguish the majority and minority spin direction. The notions of majority and minority particles always refer to the situation encountered in the bulk of the material.

${ }^{27}$ H.H. Wieder, J. Vac. Sci. Technol. 17, 1009 (1980), and other contributions in this issue.

${ }^{28}$ M.I. Dyakonov and V.I. Perel, in Optical Orientation (Ref. 7), Chap. 2.

${ }^{29}$ In $n$-type GaAs, the optically oriented electrons (now the majority carriers) are swept into the semiconductor bulk by the subsurface electric field. Hence, in principle, the semiconductor surface can become spin polarized by majority carrier transport over the Schottky barrier.

${ }^{30}$ E.H. Rhoderick and R.H. Williams, Metal-Semiconductor Contacts, Monographs in Electrical and Electronic Engineering No. 19 (Clarendon, Oxford, 1988).
${ }^{31}$ M.S. Sze, Physics of Semiconductor Devices (Wiley, New York, 1981).

${ }^{32}$ K. Sueoka, K. Mukasa, and K. Hayakawa, Jpn. J. Appl. Phys. 32, 2989 (1993).

${ }^{33}$ For a review on magneto-optical effects, see M.J. Freiser, IEEE Trans. Magn. 4, 152 (1968).

${ }^{34}$ W.B. Zeper, F.J.A.M. Greidanus, P.F. Garcia, and C.R. Fincher, J. Appl. Phys. 65, 4971 (1989); W.B. Zeper, H.W. van Kesteren, B.A.J. Jacobs, J.H.M. Spruit, and P.F. Garcia, ibid. 70, 2264 (1991). Our Pt/Co multilayers were prepared by H.W. van Kesteren, H.C. Donkersloot, and J.M. Kerkhof at the Philips Research Laboratories, Eindhoven.

${ }^{35}$ In principle, the voltage value at which the current is insensitive to a fluctuation of the optical power, could depend on the homogeneity of the power fluctuation in the optical beam and inside the GaAs tip. The optical profile of the fluctuation may not be the same for the imposed power modulation at $80 \mathrm{kHz}$ and the power modulation at $84 \mathrm{kHz}$. In other words, the measured voltage of zero IM signal $(\sim-0.2 \mathrm{~V})$ is a first order estimate for the point of zero power fluctuation sensitivity in the PM signal. However, since the measured PM signal shows a very weak dependence on voltage close to $-0.2 \mathrm{~V}$, this hardly adds uncertainty to the observation of spin-polarized tunneling.

${ }^{36}$ W. Weber, D.A. Wesner, D. Hartmann, and G. Güntherodt, Phys. Rev. B 46, 6199 (1992).

${ }^{37}$ H.-J. Drouhin, C. Hermann, and G. Lampel, Phys. Rev. B 31, 3872 (1985).

${ }^{38}$ D.T. Pierce and F. Meier, Phys. Rev. B 13, 5484 (1976).

${ }^{39}$ When an optical plane wave arrives at an abrupt interface (discrete change of refractive index from value $N_{1}$ to $N_{2}$ ), the relative Fresnel transmission coefficient for $s$-polarized light, with respect to $p$-polarized light, is given by $\rho=t_{s} / t_{p}=$ $\left[N_{2} \cos \left(\theta_{1}\right)+N_{1} \cos \left(\theta_{2}\right)\right] /\left[N_{1} \cos \left(\theta_{1}\right)+N_{2} \cos \left(\theta_{2}\right)\right]$, where $\theta_{1}$ and $\theta_{2}$ are the angles between the interface normal and the optical propagation direction in materials (1) and (2), respectively (Ref. $40)$. When the incident plane wave is circularly polarized, the ellipticity $(\epsilon)$ of the light transmitted into material (2) is given by $\sin (2 \epsilon)=2 \operatorname{Im}(\chi) /\left[1+|\chi|^{2}\right]$ (see Ref. 40), where $\chi=i \rho$. In case of an air-GaAs interface $\left(N_{1}=1, N_{2} \simeq 3.7\right.$, see Ref. 41) and an angle of incidence $\left(\theta_{1}\right)$ of $45^{\circ}$ (which yields $\theta_{2}=11^{\circ}$ ), we find that $\rho=0.83$ and $\epsilon=0.88 \times \pi / 4$. Since for circularly polarized light $\epsilon=\pi / 4$, this calculation indicates that the degree of circular polarization inside the GaAs is reduced by $12 \%$ when compared to the incident beam.

${ }^{40}$ R.M.A. Azzam and N.M. Bashara, Ellipsometry and Polarized Light (North-Holland, Amsterdam, 1987).

${ }^{41}$ D.E. Aspnes and A.A. Studna, Phys. Rev. B 27, 985 (1983). 